\title{
New capabilities at beamline 11-ID-B of the Advanced Photon Source
}

Olaf J. Borkiewicz, Uta Ruett, Kevin Beyer and Leighanne Gallington

$X$-ray Science Division, Advanced Photon Source, Argonne National Laboratory, Lemont, IL

Email Contact: borkiewicz@anl.gov

Beamline 11-ID-B has been serving user community as a dedicated total X-ray scattering beamline for over a decade, enabling a wide range of operando and in situ measurements. Over the last several month, the experimental setup within the station has undergone significant upgrades and modifications, that expand existing capabilities and introduce new methodologies, while retaining highest standards of data quality. These changes and upgrades include:

- Enabling 1-D (vertical) focusing of the beam at all operation energies and multiple focal lengths through implementation of APS-produced etched-Si compound reflective lenses

- Commissioning of a dedicated setup for grazing incidence total X-ray scattering measurements and PDF analyses (GI-PDF), enabling structural analyses on thin films and interfaces under operando conditions

- Restructuring of the entire experimental setup to enable combined total X-ray scattering and small angle scattering measurements (combined PDF/SAXS)

- Full implementation of software and hardware infrastructure to enable mail-in service at 11-ID-B 